\title{
LOS DEPARTAMENTOS DE ORIENTACIÓN EN LA REGIÓN DE MURCIA: ORIENTACIÓN Y ATENCIÓN A LA DIVERSIDAD
}

\author{
SCHOOL COUNSELLING SERVICES IN THE REGION OF MURCIA: \\ COUNSELLING AND DIVERSITY
}

\author{
Mángeles Gomariz* y Josefina Hernández Fernández** \\ Universidad de Murcia
}

\begin{abstract}
RESUMEN
El trabajo que se presenta en este artículo incluye parte de los resultados de una investigación más amplia centrada en la situación de la Orientación en los Institutos de Secundaria de la Región de Murcia. En concreto, se centra en la actuación del orientador en el área de la Orientación Vocacional, especialmente en la atención la diversidad. Para ello hemos analizado las respuestas dadas por los propios orientadores a un cuestionario elaborado para conocer la situación actual de la Orientación. Los resultados sugieren las diferencias existentes entre las propuestas oficiales o normativa actual y la realidad de los Departamentos de Orientación de la Región, a partir del gran impulso dado a los mismos por la LOGSE.
\end{abstract}

Palabras clave: Orientación Vocacional, Educación Secundaria, Atención a la diversidad, actividades de orientación.

\begin{abstract}
The work reported in this paper includes part of the results of a more extensive research focused on the Educational Guidance situation at the Secondary Schools from the Region of Murcia. Specifically, the report concentrates itself on the task of the counselor in the vocational counseling, especially in the area of diversity attention. So, we have analysed the answers of the counselors according to a questionnaire to know the actual situation. The results suggest the differences between the official
\end{abstract}

* $\quad \mathrm{M}^{\mathrm{a}}$ Ángeles Gomariz: Lic. en Pedagogía. Becaria de investigación de la Fundación Séneca, desarrolla su labor investigadora y docente en el Departamento MIDE, Facultad de Educación de la Universidad de Murcia. Sus líneas de investigación están centradas en Orientación Psicopedagógica, Orientación Vocacional y Atención a la Diversidad.

** Josefina Hernández Fernández: Profesora Titular de Orientación Educativa de la Universidad de Murcia. Ha publicado diversos libros y artículos sobre Orientación Educativa. Sus líneas de investigación se centran sobre la Orientación Vocacional, la Orientación Universitaria y la evaluación de programas de orientación. 
proposals or normative and the reality of guidance departments from Región de Murcia, after the great impulse given to them in the LOGSE.

Key words: Vocational Counseling, Secondary School, Attention to Diversity, Guidance tasks.

Actualmente, nos encontramos ante una realidad educativa que ha sufrido diversas transiciones y transformaciones, tanto estructurales, como organizativas y funcionales de una forma progresiva en los últimos diez años. Ante nosotros se presenta, por tanto, un nuevo sistema educativo implantado en escuelas e institutos que ha venido precedido e impulsado por la Ley de Ordenación General del Sistema Educativo (L.O.G.S.E., 1990). Es un hecho que el sistema educativo actual está plenamente establecido en los centros, pero la situación actual, en la que se camina hacia la mejora y calidad del sistema educativo (recordemos la Ley de Calidad del Sistema Educativo, en proyecto), nos invita a reflexionar e investigar los elementos que hacen posible su desarrollo en la práctica.

En este trabajo presentamos una síntesis del estudio empírico que hemos realizado con el propósito de conocer la situación actual de la Orientación en los Institutos de Educación Secundaria (I.E.S.) de la Región de Murcia. En concreto, nos interesa profundizar en los cambios organizativos que ha experimentado el sistema educativo actual, y la Orientación como parte del mismo, así como conocer de qué manera se están desarrollando estos cambios en la práctica, a partir de la opinión de los orientadores que componen los Departamentos de Orientación de los centros de Enseñanza Secundaria.

\section{Contextualización del estudio}

Varias son las razones que nos han movido a centrar la investigación en la etapa de Educación Secundaria.

En primer lugar, encontramos en ella la prolongación de la escolaridad obligatoria hasta los 16 años, lo que supone, de acuerdo con Álvarez y Rodríguez (2000), una situación nueva que introduce por primera vez en la historia educativa de España la L.O.G.S.E. (1990). Para Esteve (2000), al declarar obligatoria la Enseñanza Secundaria, la finalidad propedéutica tradicional de la misma como paso hacia la Universidad, deja paso a una etapa educativa con valor en sí misma, con carácter terminal. En este sentido, la Educación Secundaria se vincula estrechamente al contexto social y a la realidad laboral de los alumnos. Una formación general, básica y flexible como la que aporta esta etapa será útil en la medida en que permita al adolescente adaptarse mejor y más rápidamente a las necesidades y demandas continuamente cambiantes del entorno social y laboral. Es decir, su sentido más profundo es preparar a los adolescentes para que se conviertan en verdaderos ciudadanos con participación activa en la sociedad (Blanco, 1995). Como consecuencia de esta ampliación de la escolaridad obligatoria aparece una mayor heterogeneidad de alumnos que acceden y completan sus estudios de Educación Secundaria. Este hecho, no exento de dificultades, exige una respuesta adaptada a las necesidades de cada uno de nuestros alumnos, es decir, se precisa de una atención a la diversidad de alumnado que recibe formación en Enseñanza Secundaria.

En segundo lugar, la progresiva ampliación del abanico de posibilidades formativas ha sido consecuencia, en parte y según la opinión de Álvarez y Rodríguez (2000), de la si- 
tuación actual de impredicibilidad y variación de las necesidades del sistema productivo. Entre los instrumentos que la L.O.G.S.E. propone para dar respuesta a las diferentes capacidades, intereses y motivaciones para aprender de cada uno de los alumnos, destaca la aplicación práctica del derecho a la Orientación. En la parte III, punto 9.12.5 del Proyecto para la Reforma ya encontramos una primera referencia a la necesidad de creación de los servicios de Orientación en Educación Secundaria: "Esta búsqueda de un equilibrio entre comprensividad y respeto a las diferencias (...) de los estudiantes, elemento crucial para un correcto planteamiento de la Educación Secundaria, exige la puesta en marcha de unos servicios eficaces de orientación psicopedagógica».

En último lugar, constatamos la generalización del Departamento de Orientación en los centros de Educación Secundaria. El impulso de la Orientación a través de la organización de recursos materiales, humanos y económicos ha sido uno de los objetivos principales perseguidos por la Reforma educativa promovida a partir de la promulgación de la L.O.G.S.E. (1990). El D.O. se constituye, desde la perspectiva de la normativa actual, en un órgano de coordinación docente que ha de colaborar en la planificación y desarrollo de medidas de atención a la diversidad de capacidades, motivaciones e intereses de los alumnos. Desde este espíritu y partiendo de las funciones que se le atribuyen, las propuestas de actuación del D.O. se desarrollan en torno a tres grandes ámbitos que extraen de la legislación numerosos autores (Babío, 1996; Hortelano y Jorge, 1997; Bisquerra, 1998; Vélaz de Medrano, 2000):

- Apoyo al proceso de enseñanza-aprendizaje.

- Apoyo y desarrollo del Plan de Acción Tutorial (P.A.T.).

- Apoyo en la organización y desarrollo del Plan de Orientación Académica y Profesional (P.O.A.P.).

Estos ámbitos no son competencia exclusiva del D.O. ni pueden llevarse a cabo de forma solitaria por el Psicopedagogo. Por ello, opinamos junto con Hortelano y Jorge (1997), que las intervenciones orientadoras se deben dirigir a la coordinación y apoyo al centro y entenderse el D.O. como equipo de trabajo. En palabras de Babío (1996:448) «es importante que la normativa de funcionamiento del centro regule las relaciones entre los distintos órganos de coordinación y posibilite el desarrollo de las distintas actividades desde un trabajo colaborativo».

Consideramos que la labor del Psicopedagogo dentro de un D.O., en línea con lo que expresa la normativa educativa actual y, de acuerdo con la opinión de Guerrero (1995), se va a desarrollar prioritariamente en dos ámbitos: la Orientación Educativa y Profesional y la Atención a la Diversidad. Este último ámbito de actuación ocupa mucho más tiempo de lo que se puede pensar y constituye uno de los pilares de intervención del D.O. de un centro de Educación Secundaria. De aquí se deriva el interés de nuestro estudio por establecer una conexión entre estos dos ámbitos de actuación prioritaria de los D.O., centrándonos en analizar si existen actuaciones de Orientación Vocacional y Profesional diferenciadas en función de la diversidad de alumnado al que se dirigen.

Aunque algunos autores se refieran a los alumnos que forman parte de la Diversidad como «alumnos de Educación Especial», nosotras preferimos denominarlos como alumnos con necesidades educativas, en función de la terminología más actual que, por otra parte, nos resulta más integradora. Entendemos la diversidad como un aspecto, una variable inherente a la condición humana (Fierro, 1985). O bien, como indica López Melero (1995:27), la diversidad es «lo más genuinamente natural del ser humano». Por tanto, debemos reco- 
nocer que todos los alumnos tienen necesidades educativas y todos demandan una atención individualizada de la escuela y de los diferentes agentes que intervienen en su educación.

Siguiendo a Fierro (1985) podemos afirmar que todas las necesidades educativas son especiales, pues son propias de determinados alumnos. Lo que ocurre es que algunas de ellas están más generalizadas, y afectan a un mayor número de alumnos, y otras son minoritarias. Por tanto, el concepto de diversidad se puede representar en un continuo. No sólo se refiere al hándicap, como señala López Melero (1995), sino que incluye a todos los colectivos menos favorecidos de la sociedad que por diversas causas pueden situarse en algún punto de ese continuo. Todos y cada uno de nosotros formamos parte de la diversidad, puesto que en cualquier momento de nuestra vida y en especial de nuestra trayectoria educativa, podemos requerir respuestas adaptadas a nuestras necesidades.

La idea de necesidades educativas que defendemos no se centra en lo que el alumno es, sino en su interrelación con el medio escolar. De aquí que nuestro interés se concentre en nuestra investigación en lo que la escuela hace con cada uno de sus alumnos. Es decir, nos interesa conocer cuál es la intervención educativa y, específicamente, orientadora que se desarrolla con estos alumnos en los I.E.S. participantes en nuestro estudio. Para conseguir datos relevantes, decidimos dividir el continuo de la Diversidad en los siguientes grupos de alumnos, atendiendo a la realidad actual que encontramos en los centros educativos de Secundaria: alumnos con dificultades de aprendizaje; futuros alumnos de Programas de Garantía Social; alumnos con necesidades educativas especiales; alumnos de minorías; alumnos con dificultades de adaptación socioafectiva.

Nuestra intención, al adoptar este agrupamiento, no ha sido etiquetar o categorizar a los alumnos en función de sus necesidades. Muy al contrario, la finalidad de este agrupamiento tiene un carácter práctico, en dos vertientes. En primer lugar, el criterio para la agrupación ha sido observar la realidad práctica de la Atención a la Diversidad en los centros de Educación Secundaria. Pensamos que conocer las distintas fuentes de diversidad del alumnado que cursa estudios de Secundaria y la atención que se le presta, nos puede ayudar a desarrollar estrategias óptimas para intervenir en la diversidad respetando las diferencias individuales. Es decir, atendemos al criterio de atención e intervención diversificada e individualizada que requiere cada uno de nuestros alumnos, por parte de la Orientación. En segundo lugar, buscamos cierta eficacia en la recogida de información. Es decir, pretendemos obtener la mayor cantidad de información referida a cada uno de los grupos de alumnos con necesidades, y que ésta no se disuelva en la generalidad de todos ellos.

La exposición de estos motivos nos lleva a concluir sobre la idoneidad de plantear nuestro estudio de la Orientación y la Atención a la Diversidad como ámbito prioritario de intervención, y su desarrollo actual en la etapa de Educación Secundaria Obligatoria (E.S.O.).

\section{Metodología}

\section{Propósito y objetivos}

El presente estudio descriptivo se enmarca en el seno de una investigación más amplia cuya finalidad consiste en desarrollar una respuesta orientadora adaptada a la diversidad del alumnado que encontramos en los centros de E.S.O. de la Región de Murcia. 
De esta investigación desglosaremos y analizaremos en este estudio aquellos aspectos que nos permitan conocer cómo se organiza el D.O. en los centros de E.S.O. de la Región de Murcia, y cuáles son las actuaciones de Orientación Vocacional y Profesional que se desarrollan en los mismos, con una especial incidencia en el papel que desempeña la Orientación en la Atención a la Diversidad. Pretendemos, de esta manera, obtener una visión general acerca de dos realidades presentes en los centros de Educación Secundaria, que se implican mutuamente: el D.O., como órgano encargado de la intervención psicopedagógica, por un lado; y la Atención a la Diversidad como un ámbito de actuación prioritaria en los centros de Secundaria desde la implantación de la L.O.G.S.E.

Para alcanzar dicha finalidad nos proponemos los siguientes objetivos específicos:

1. Conocer las características de los centros de Educación Secundaria de la Región de Murcia.

2. Explorar la estructura y composición de los Departamentos de Orientación en los centros de Educación Secundaria de la Región de Murcia.

3. Determinar si existen alumnos con necesidades educativas especiales en los centros de Educación Secundaria y a qué grupos pertenecen.

4. Observar si existen medidas extraordinarias de Atención a la Diversidad en los centros de Secundaria de la Región de Murcia.

5. Explorar las actuaciones, actividades o programas de Orientación Vocacional y Profesional que aparecen reflejadas en la programación del Departamento de Orientación.

6. Determinar si existen actividades o intervenciones de Orientación diferenciadas en función de la diversidad de alumnado que forma parte de los centros de Educación Secundaria de la Región de Murcia.

\section{Población y Muestra}

El universo de estudio está compuesto por todos los centros de Educación Secundaria de la Región de Murcia. La población a la que se dirige nuestro estudio es la totalidad de I.E.S. de la Región de Murcia en los que se imparte algún curso de $2^{\circ}$ ciclo de E.S.O. $\left(3^{\circ} \mathrm{y} / \mathrm{o} 4^{\circ} \mathrm{de}\right.$ E.S.O.) y que cuentan con D.O., durante el curso escolar 1998/99. Dentro de la población se incluyen centros públicos y privados. Además se cuenta con los I.E.S. que imparten enseñanzas L.O.G.S.E. exclusivamente (de reciente creación), y centros que pueden combinar en sus aulas enseñanzas L.O.G.S.E. (Secundaria Obligatoria, Bachilleratos, Ciclos Formativos, Diversificación, Garantía Social) con enseñanzas a extinguir (Bachillerato Unificado Polivalente-B.U.P., Curso de Orientación Universitaria-C.O.U. y/o Formación ProfesionalF.P.). Por tanto, y atendiendo al ámbito geográfico de la Región de Murcia, contamos con una población de 99 centros educativos de Enseñanza Secundaria.

En el proceso de selección de la muestra hemos utilizado un muestreo mixto (Hernández Pina y otros, 1995). Así, en un primer momento se ha realizado un muestreo no probabilístico y, dentro de esta categoría, lo podemos considerar como un muestreo intencional u opinático (Arnal, Rincón, y Latorre, 1994), estableciendo determinados criterios para seleccionar los centros. 
Una vez obtenida la muestra inicial, se procedió a considerar a todos los centros que la componían como muestra invitada de nuestra investigación. En este momento se empleó un muestreo aleatorio simple, que cumple con el principio de probabilidad, imprescindible para obtener una muestra final lo más representativa posible de la población.

Finalizado el proceso de selección de la muestra, contamos con 88 centros que forman parte de nuestra muestra invitada y un total de 52 centros participantes en el estudio (muestra real o productora de datos).

\section{Procedimiento y descripción del instrumento}

Para poder cubrir tanto los objetivos previstos para este estudio, como los que se derivan de la investigación general, se ha recurrido a la metodología de encuesta o cuestionario, que tal y como opinan Buendía y otros (1997: 120) es «capaz de dar respuesta a problemas tanto en términos descriptivos como de relación de variables (...)».

Como destinatario del cuestionario se fijó al Orientador/a que desempeña su tarea profesional en un centro de Educación Secundaria. Para dar respuesta a los objetivos propuestos, y teniendo en cuenta el ámbito y los sujetos a los que iba dirigido, se elaboró un cuestionario que en una primera versión constaba de 58 preguntas. Este instrumento comienza a construirse a partir de la información obtenida de las distintas entrevistas realizadas, la bibliografía consultada y los objetivos de investigación propuestos inicialmente.

Para proceder a la depuración y perfeccionamiento del cuestionario piloto, se procedió a través del sistema de juicio de expertos. Entregamos el cuestionario a un reducido grupo de profesionales del ámbito de la educación que trabajan en los siguientes campos: Servicio de Inspección Educativa, Equipos de Orientación y Evaluación Psicopedagógica (E.O.E.P.s), Institutos de Educación Secundaria, Departamentos de Orientación, Programas de Garantía Social, especialistas en Diagnóstico Psicopedagógico, especialistas en Orientación. Este grupo, formado por ocho personas, evaluó la calidad formal del instrumento, valorando prioritariamente aspectos tales como la adecuación del cuestionario a los objetivos perseguidos por la investigación, claridad en la formulación de las cuestiones y su ajuste a la realidad educativa del momento actual.

Fruto de todo ello es el cuestionario definitivo aplicado en la investigación en la que se apoya el presente estudio. Dicho cuestionario se compone finalmente de 54 preguntas, de las que 39 son cerradas y 15 abiertas. Las cuestiones cerradas, a su vez, pueden ser, bien de respuesta múltiple, bien de tipo escala Likert.

La composición del cuestionario empleado, de acuerdo con las categorías o dimensiones de los datos que pretendemos recoger, se distribuye en cinco grandes bloques: datos de identificación del centro; datos educativos del centro; características del D.O.; actuaciones de Orientación en el centro; centros con Programa de Garantía Social (P.G.S.).

Una vez seleccionada la muestra de la investigación, se procedió a la aplicación del cuestionario. Se pensó en un modo de entrega del cuestionario que resultara lo más personalizado posible, a fin de evitar al máximo la «muerte muestral». Optamos por un proceso organizado en tres fases diferenciadas:

$1^{\text {a) }}$ Contacto telefónico con el Orientador/a de cada Instituto de Educación Secundaria. En esta breve conversación se explicaba el propósito de la investigación a nuestros 
destinatarios y se concretaba una fecha próxima para hacerle entrega del cuestionario en mano.

$2^{a}$ ) Visita a los centros para proceder a la entrega personal del cuestionario al Orientador/a.

$3^{a}$ ) Finalmente, el cuestionario se entregaba con un sobre y un sello para que el Orientador nos lo devolviera por correo. Esta opción facilitaba que las respuestas al cuestionario fueran más pausadas y personales que si se contestaba en presencia de la investigadora. Además facilitaba también la ardua tarea que suponía el recoger los cuestionarios personalmente, de la misma manera que se entregaron.

Conforme se recibían los cuestionarios, se iba desarrollando el largo proceso de categorización de variables, para poder realizar posteriormente su procesamiento estadístico. Una vez que dispusimos del total de cuestionarios se inició el análisis de datos, que hemos realizado con el programa estadístico SYSTAT, versión 5.0 para Windows.

\section{Análisis de resultados}

\section{Objetivo 1: Conocer las características de los centros de Educación Secundaria de la Región de Murcia}

Los 52 I.E.S. que componen nuestra muestra participante proceden de todas las zonas de la Región de Murcia. El 48\% de los Institutos que conforman nuestra muestra se reparte entre Murcia capital (12 centros) y los municipios de Cartagena (6 centros) y Lorca (4 centros). El 52\% restante se distribuye entre otros 20 municipios participantes, a razón de 1, 2 ó 3 centros por población.

En cuanto a la ubicación del centro dentro de la localidad a la que pertenece, el $56 \%$ de los centros están situados en los alrededores de la ciudad, mientras que el resto tiene una ubicación céntrica.

Un aspecto interesante a destacar es el tipo de enseñanzas que se imparten en los I.E.S. objeto de nuestro estudio. En el momento de aplicación del cuestionario, nos encontramos en la recta final de la implantación de la L.O.G.S.E. Por ello, algunos de los centros de la muestra combinan en sus aulas enseñanzas de la nueva secundaria con enseñanzas a extinguir, bien del B.U.P. (Bachillerato Unificado Polivalente) o bien de F.P. (Formación Profesional.). La distribución, por tanto, de esta variable en nuestra muestra queda como se observa en la figura 1.

En concreto, el $40,38 \%$ de los centros participantes imparten $1^{\mathrm{er}}$ y $2^{\circ}$ ciclo de E.S.O., mientras que casi el $60 \%$ imparte exclusivamente el $2^{\circ}$ ciclo (Fig. 2 ). Como podemos observar, la mayor parte de los centros imparte solo el $2^{\circ}$ ciclo de E.S.O., pues existen numerosos centros de Primaria que han incorporado en su oferta educativa el $1^{\text {er }}$ ciclo de Secundaria $\left(1^{\circ}\right.$ y $2^{\circ}$ de E.S.O.).

Un dato a destacar es que la media del número de grupos en $1^{\circ}$ de E.S.O., para los centros que imparten ambos ciclos es de 1,4. Destaca un 38,10\% de los centros (8) que posee 3 líneas de este primer curso. La media del número de grupos para $2^{\circ}$ curso de E.S.O. en los mismos centros aumenta hasta un 1,7. También en este caso sobresale un 33,33\% de centros que tiene 3 líneas en $2^{\circ}$ curso de Educación Secundaria. 


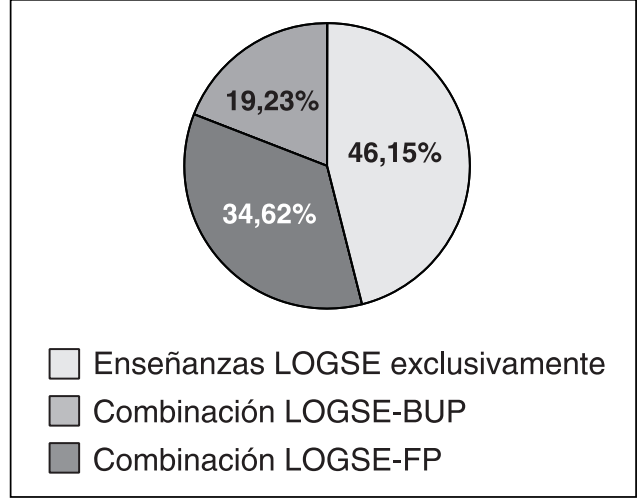

FIGURA 1. Centros de enseñanzas que imparten.

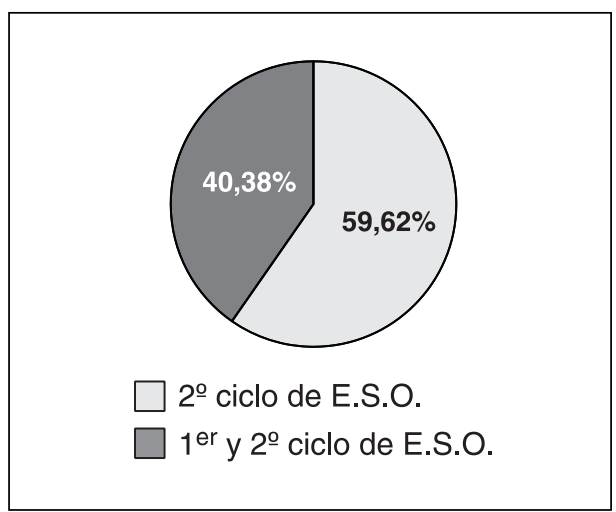

FIGURA 2.

Centros y ciclos que imparten.

En cuanto al $2^{\circ}$ ciclo de E.S.O., encontramos que por lo general existe un mayor número de grupos de $3^{\circ}$ y $4^{\circ}$ curso de E.S.O. en los centros que imparten exclusivamente el $2^{\circ}$ ciclo. Más de la mitad de estos centros $(51,61 \%)$ tienen entre 6 y 7 grupos de $3^{\text {er }}$ curso. La mayor parte de estos centros, un 48,39\%, posee entre 3 y 6 grupos de $4^{\circ}$ curso. Destaca, asimismo, que 10 de los 31 centros que componen este grupo imparte enseñanzas únicamente en $3^{\circ}$ de E.S.O., siendo además centros que no poseen el $1^{\mathrm{er}}$ ciclo de Secundaria Obligatoria.

Para finalizar con la descripción de la muestra, podemos aportar el perfil característico de los centros que han participado en nuestro estudio.

Instituto de Educación Secundaria Obligatoria, situado en la periferia de una localidad urbana de la Región de Murcia que, durante el curso 1998/99, ha impartido fundamentalmente enseñanzas de $2^{\circ}$ ciclo de E.S.O., con un número de líneas que se aproxima a 6 en tercer curso y oscila entre 3 y 6 en cuarto curso, que posee Programa de Diversificación Curricular, atiende a alumnos con necesidades educativas especiales y dispone de Departamento de Orientación, cuyo coordinador es el Psicopedagogo.

\section{Objetivo 2. Conocer la estructura y composición de los Departamentos de Orienta-} ción en los centros de Educación Secundaria de la Región de Murcia

\section{Antigüedad del Departamento de Orientación en Educación Secundaria}

Atendiendo a la variable antigüedad de D.O. en el centro, la media se sitúa en 5,5 años, durante el curso 1998/99, encontrando el mínimo en un año y el máximo en doce.

En cuanto a la antigüedad del Psicopedagogo en el centro, un 38,46\% de los encuestados llevaban un año en el mismo centro. En la mayor parte de los centros, el Psicopedagogo ha permanecido entre uno y tres años en el mismo Instituto (el 65,38\% de la muestra).

La antigüedad en la creación del D.O. y en la permanencia del Psicopedagogo en dicho centro, coincide en 15 casos $(28,85 \%$ de los centros de la muestra). 
El máximo de años de permanencia del Psicopedagogo en un mismo centro se sitúa en 10, coincidiendo en este caso 3 Psicopedagogos en centros cuyo D.O. fue creado hace 10 años.

Por último, destacamos la situación administrativa del Psicopedagogo que en los centros participantes en la investigación se concreta en los siguientes datos:

- El 65,38\% estaba en su destino definitivo, más de la mitad de los cuales (el 38,46\%) tenían una antigüedad no superior a 3 años en ese centro.

- Un 30,77\% estaban en situación de interinidad, de los que la mayoría (el 21,15\%) sólo llevan un año en el mismo centro.

- Tan sólo $1(1,92 \%)$ se encontraba con plaza pero sin el destino definitivo.

Observamos en estos datos que existe una gran variedad en la movilidad de los Técnicos de Orientación en los centros de la muestra, siendo menor, como es lógico, en los que adquieren el destino definitivo. Es decir, la mayor o menor movilidad de los Orientadores está determinada, en muchos casos, por la situación administrativa del Técnico. Esta problemática, que nosotros hemos constatado empíricamente en la Región de Murcia, tiene su paralelismo en otras comunidades autónomas que tienen competencias en educación desde hace algunos años, como constatan las aportaciones realizadas por Rodicio y otros (2001) sobre la situación de la Orientación en Galicia.

Sintetizando, podemos afirmar que si bien algún D.O. fue creado como fruto de la normativa de 1987, la creación de la mayoría de ellos responde al impulso dado por la L.O.G.S.E., a la implantación de los Departamentos de Orientación en Secundaria, bien en centros ya establecidos, bien en los de nueva creación.

\section{Componentes del Departamento de Orientación}

En cuanto a los componentes del D.O., encontramos que en el $100 \%$ de los centros existe la figura del Psicopedagogo, lo que no es significativo ya que era condición previa de nuestra selección muestral, pues es el destinatario del cuestionario empleado en la recogida de información.

Sin embargo, no en todos los centros el Psicopedagogo es el Jefe del D.O., aunque sí en la mayoría, concretamente en el $86,54 \%$ de los centros. En el 9,62\% de los centros de la muestra el Jefe del D.O. es un Profesor de Ámbito (Científico-Tecnológico, Socio-Lingüístico o del Área Práctica).

Con relación al resto de componentes del D.O., nos encontramos con el profesor de Ámbito Científico-Tecnológico en un 69,23\% de casos; el profesor de Ámbito SocioLingüístico en un 65,38\% y el profesor del Área Práctica en un 55,77\% de los centros.

Destaca el dato de que la mayoría de centros que tienen en el D.O. algún profesor de Ámbito, tanto Científico-Técnico, como Socio-Lingüístico, como del Área Práctica, son centros que imparten exclusivamente enseñanzas L.O.G.S.E.

En todos los centros en los que hay profesor de Ámbito Socio-Lingüístico existe también profesor de Ámbito Científico-Tecnológico, lo que sucede en un 65,38\% de los centros. A este porcentaje se añade el 3,85\% de los centros en los que el único profesor de Ámbito que forma parte del Departamento de Orientación es el de la especialidad CientíficoTecnológica. 
El profesor de Formación y Orientación Laboral (F.O.L., destinado a los Ciclos Formativos y al Programa de Garantía Social) está presente en el 21,15\% de los centros. El Maestro de Pedagogía Terapéutica (P.T., o Profesor de Apoyo en lenguaje coloquial) aparece en un 53,85\% de los casos. Mientras que el Maestro de Audición y Lenguaje (A.L. o Logopeda), forma parte del Departamento de Orientación en un 15,38\% de los centros de la muestra. Es decir, tan sólo en 8 centros de los 47 que afirman tener escolarizados alumnos con necesidades educativas especiales existe Maestro especialista en Audición y Lenguaje. La figura del profesor de Educación Compensatoria forma parte del D.O. de los centros encuestados en un 19,23\% de los mismos. El Fisioterapeuta, como especialista en la atención al alumno con necesidades de tipo físico o motor, aparece en el 3,85\% de los centros participantes. Otros profesionales que aparecen aisladamente en algunos centros son:

- Asistente o Trabajador Social: 3 centros.

- Auxiliares técnicos educativos: 2 centros.

- Profesor técnico de Servicios a la Comunidad: 2 centros.

- Maestro de Garantía Social (Área de Formación Básica): 1 centro.

- Intérprete de Lengua de Signos: 1 centro.

Los resultados presentados nos permiten apreciar que la figura del Psicopedagogo, Orientador o Técnico en Orientación es sobre la que se organiza el órgano de coordinación y asesoramiento que constituye el Departamento de Orientación de un centro de Educación Secundaria. En cuanto al profesorado de Ámbito, los datos nos hacen pensar que el más arraigado es el Profesor del Ámbito Científico-Tecnológico.

Sin embargo, encontramos que la aparición del resto de componentes que ha de formar el staff del Departamento de Orientación, no es sistemática en todos los centros. Los profesionales que aparecen en menor proporción se relacionan directamente con el ámbito de Atención a la Diversidad. Por un lado, la presencia de Maestros de Apoyo a la Integración de los alumnos con necesidades educativas especiales (Pedagogía Terapéutica, Audición y Lenguaje, Fisioterapeuta, Asistente Social, Intérprete de Lengua de Signos) no está generalizada en los Departamentos de Orientación de los centros de la muestra. Por otro lado, tampoco los Profesores que han de colaborar en los Programas de Diversificación y los Programas de Garantía Social (Profesores de Ámbito, Maestros de Formación Básica, Formación y Orientación Laboral) aparecen en muchos de los centros que desarrollan estos programas.

\section{Objetivo 3: Determinar si existen alumnos con necesidades educativas especiales en los centros de Educación Secundaria y a qué grupos pertenecen}

\section{Alumnos con necesidades educativas especiales}

En primer lugar, podemos decir que un centro atiende a la Diversidad si en él cursan estudios alumnos con diferentes necesidades educativas. A este respecto, de los 52 centros participantes en este estudio, podemos decir que 47 acogen en sus aulas alumnos con necesidades educativas especiales (a.c.n.e.e., a partir de aquí), lo que supone un 90,38\% de la muestra (Fig. 3). Entre los 5 centros que no tienen escolarizados a.c.n.e.e., uno de ellos imparte exclusivamente nuevas enseñanzas, mientras que los cuatro restantes simultanean enseñanzas de la nueva Secundaria con el anterior plan de Bachillerato (B.U.P.). 


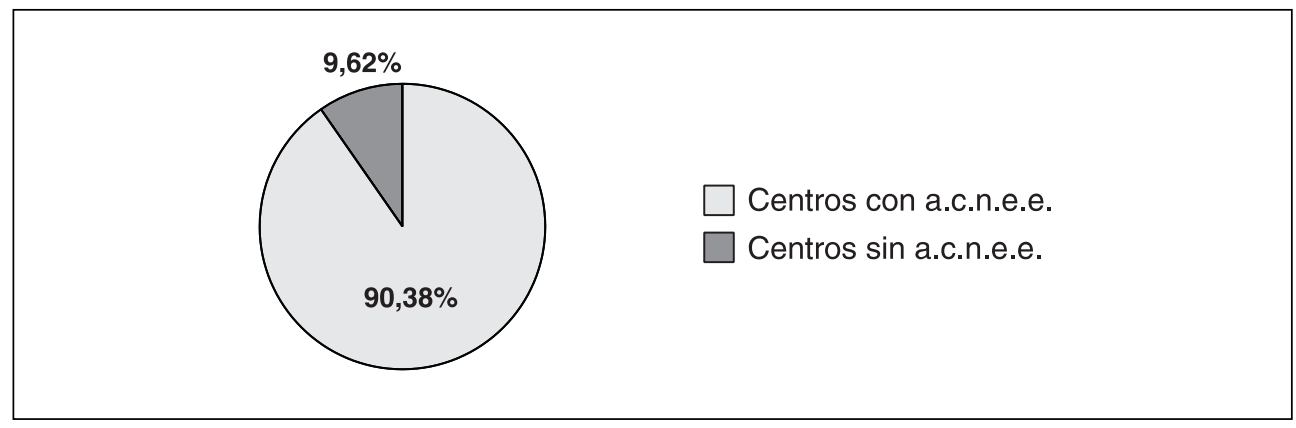

FIGURA 3.

Centros con atención a alumnos con necesidades educativas especiales.

En cuanto a la especificación de estas necesidades educativas especiales, la mayor parte de los centros afirma acoger alumnos con necesidades educativas permanentes (un $75 \%$ de la muestra así lo señala) y alumnos con dificultades de aprendizaje, lo que expresa un $78,85 \%$ de los I.E.S. participantes. También existen alumnos con necesidades de compensación sociocultural (magrebíes, minorías étnicas, ...) que aparecen en 4 de cada 10 centros de la muestra. Por último, encontramos que el $25 \%$ de los Institutos afirma tener escolares con dificultades de adaptación socio-afectiva.

Contrastando la normativa legal con la realidad reflejada en nuestros datos, se observa, que aquélla es respetada por la práctica totalidad de la muestra. Es decir, la mayoría de los centros de la muestra son centros de integración, pues acogen en sus aulas a a.c.n.e.e., pudiendo atribuir las excepciones a circunstancias en parte justificadas por razones organizativas, como pudiera ser el proceso de implantación de las enseñanzas promovidas por la L.O.G.S.E. que no se había completado en el momento de realizar el estudio.

Sin embargo, si comparamos la existencia de Maestros de Apoyo a la Integración, que han de formar parte del Departamento de Orientación, y los alumnos con necesidades educativas especiales escolarizados en los centros de la muestra, encontramos una clara discrepancia. Es decir, en más de la mitad de los centros que atienden a alumnos con necesidades educativas, no existen Maestros especialistas que desarrollen su labor de Atención a la Diversidad.

\section{Objetivo 4. Observar si existen medidas extraordinarias de Atención a la Diversidad en los centros de Secundaria de la Región de Murcia}

\section{Programas de Diversificación Curricular}

Aproximadamente el $70 \%$ de la muestra (36 centros) incluye en su oferta educativa un Programa de Diversificación Curricular (P.D.C.), como medida de respuesta a la diversidad (Fig. 4). De estos 36 centros, 22 son de enseñanza L.O.G.S.E. Es decir, un solo centro L.O.G.S.E. no posee P.D.C., mientras que el resto ya lo tiene organizado. Sin embargo, en los centros que combinan enseñanzas L.O.G.S.E. y B.U.P. la proporción se invierte. Hay más institutos que no poseen P.D.C. (13 centros) que los que ya lo tienen ( 5 centros). 


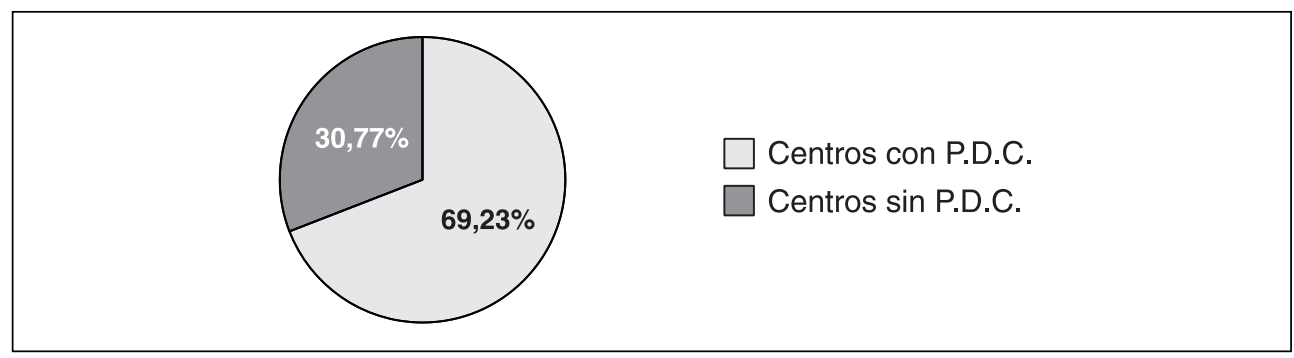

FIGURA 4.

Programa de Diversidad Curricular.

En relación con la antigüedad del P.D.C. en los centros que lo poseen, podemos afirmar que más de la mitad (58\%) lo tienen implantado entre 2 y 4 años y un $33 \%$ indica que la antigüedad de este programa en el centro es de 5 a 7 años.

Otro dato a resaltar es el número de alumnos que forma parte del P.D.C. en los centros de la muestra. La media de alumnos se sitúa en torno a 12, llegando algunos centros a tener más de 30 alumnos de Diversificación, e incluso aparece un Instituto que los sitúa en torno a los 50 alumnos. Estas cantidades pueden deberse a la existencia de más de un curso de Diversificación.

En cuanto a la existencia de profesorado de Apoyo a los Ámbitos del Departamento de Orientación que debe impartir las áreas específicas del P.D.C., encontramos los siguientes resultados:

- De los 36 centros que poseen P.D.C., sólo 2 no disponen de Profesor del Ámbito Científico-Tecnológico que pertenezca al D.O., lo que supone un 3,85\% del total de centros de la muestra y un 5,56\% del total de centros que imparten P.D.C.

- En el caso del Profesor del Ámbito Socio-Lingüístico, aumenta la proporción de centros que cuentan con P.D.C. y, sin embargo, no disponen de este Profesor. En concreto, nos encontramos con 4 de los 36 centros de la muestra que imparten un P.D.C. y que no disponen de este profesor. Lo cual se traduce en un $11,11 \%$ del total de centros con P.D.C. y un 7,69\% con relación al total de centros participantes en el estudio.

\section{Programas de Garantía Social}

En cuanto a los Programas de Garantía Social (P.G.S.), que constituyen una parte importante de la investigación general en la que se enmarca este estudio, encontramos que el $28,85 \%$ de los Institutos participantes imparten algún P.G.S. Es decir, 15 de los 52 centros participantes incluyen en su oferta educativa estos programas encaminados a la transición e inserción en el mundo laboral de los jóvenes (Fig. 5).

Más de la mitad de estos centros (8) se concentran en los I.E.S. de la muestra que imparten únicamente las enseñanzas promovidas por la L.O.G.S.E.

Si nos centramos en la especialidad de F.O.L., que debe formar parte del equipo educativo de un P.G.S., observamos una clara contraposición de la realidad con la normativa. En concreto, de los 15 centros de Secundaria que imparten algún P.G.S., tan sólo 4 tienen Pro- 


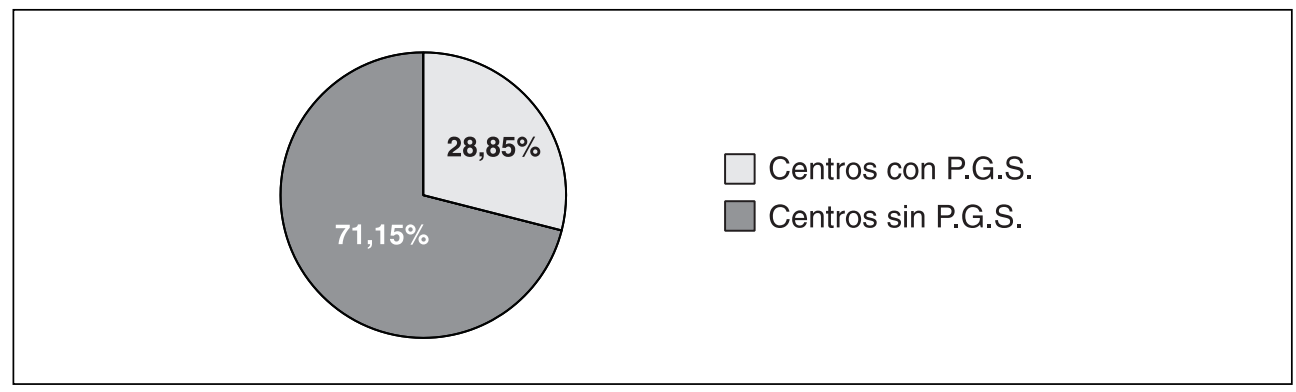

FIGURA 5.

Programa de Garantía Social.

fesor de F.O.L., que forme parte del D.O. Lo que supone un $26,65 \%$ del total de centros con P.G.S.

En la mayoría de centros (9 de 15) se imparte un único P.G.S. En 5 centros se imparten 2 especialidades simultáneamente y en un centro se llega a impartir 3 modalidades distintas de P.G.S., al mismo tiempo.

Destacamos, además, que todos los centros que poseen Programa de Garantía Social tienen Programa de Diversificación Curricular, excepto uno. Esto se explica porque la Diversificación Curricular se sitúa como una medida de atención a la diversidad prioritaria a la derivación del alumno a un Programa de Garantía Social.

Sintetizando este aspecto, podemos destacar que existe gran diferencia entre lo marcado por la normativa vigente para estos Programas y lo que está siendo su desarrollo en la práctica. Lo más significativo es que los profesores encargados de impartir, según la legislación, tanto los Programas de Diversificación como los de Garantía Social, no aparecen en la mayoría de los centros que incluyen dichos Programas en su oferta educativa.

\section{Objetivo 5. Explorar las actuaciones, actividades o programas de Orientación Vocacional y Profesional que aparecen reflejadas en la Programación del Departamento de Orientación.}

En este apartado vamos a realizar una presentación más cualitativa de los datos, que en apartados anteriores, centrándonos en las actividades de Orientación Vocacional y Profesional que se desarrollan, con mayor frecuencia, para el $1^{\mathrm{er}}$ y $2^{\circ}$ ciclo de E.S.O.

En primer lugar, observamos que, independientemente del tipo de intervenciones, se llevan a cabo un mayor número de actuaciones de Orientación Vocacional y Profesional en $2^{\circ}$ ciclo de E.S.O., con respecto al $1^{\text {er }}$ ciclo. Se da una media de 9,6 actuaciones de Orientación en $2^{\circ}$ ciclo de E.S.O., mientras que en el $1^{\circ}$ la media descriptiva desciende hasta 1,71, diferencia que resulta significativa con un margen de confianza del $95 \%$

A continuación, presentamos las intervenciones orientadoras que desarrollan los Departamentos de Orientación en mayor medida, en $1^{\text {er }}$ y $2^{\circ}$ ciclo de E.S.O. Para realizar la gradación nos basamos en las categorías donde se han concentrado las mayores frecuencias del total de respuestas facilitadas por los Orientadores (Tabla 1). 


\section{TABLA 1.}

\begin{tabular}{|c|c|}
\hline $1^{\text {er }}$ ciclo de E.S.O. & $2^{\circ}$ ciclo de E.S.O. \\
\hline 1. Fomento de las Habilidades Sociales. & 1. Información Académica y Vocacional. \\
\hline $\begin{array}{l}\text { 2. Información Académica (optativas, } \\
\text { posiblesitinerarios) y Vocacional. }\end{array}$ & 2. Evaluación de Actitudes e Intereses. \\
\hline 3. Evaluación de Capacidades y Aptitudes. & 3. Evaluación de Capacidades y Aptitudes. \\
\hline 4. Desarrollo del Autoconcepto. & 4. Toma de Decisiones. \\
\hline 5. Dinámicas para el Autoconocimiento. & 5. Información sobre formación no reglada. \\
\hline & 6. Dinámicas para el Autoconocimiento. \\
\hline & 7. Información Laboral. \\
\hline & 8. Desarrollo del Autoconcepto. \\
\hline
\end{tabular}

Comparando las intervenciones en ambos ciclos, apreciamos que se da una mayor relevancia en el $1^{\text {er }}$ ciclo a las actividades relacionadas con el área de Orientación para el Desarrollo Personal y Social, con respecto al $2^{\circ}$ ciclo. Sin embargo, en $2^{\circ}$ ciclo predominan las intervenciones de Información Académica y Vocacional, Información Laboral, Evaluación de capacidades e intereses, Actividades para el desarrollo del proceso de Toma de Decisiones, todas ellas consideradas como parte del área de Orientación para el Desarrollo de la Carrera y Orientación Profesional.

Esta diferenciación en las actividades de Orientación programadas para ambos ciclos es totalmente compatible con la función de proporcionar Orientación a todos los alumnos que se establece para el Psicopedagogo. Además, refleja la preocupación por atender a la diversidad de necesidades que presentan los alumnos en los diferentes momentos de su trayectoria educativa y su desarrollo vocacional.

\section{Objetivo 6. Determinar si existen actividades o programas de Orientación diferenciados en función de la diversidad de alumnado que forma parte de los centros de Educación Secundaria.}

A continuación vamos a describir, para cada uno de los grupos de alumnos que presentan necesidades diferenciadas, las intervenciones orientadoras que desarrollan los Departamentos de Orientación en mayor y menor proporción. Para realizar la gradación nos basamos en las categorías donde se han concentrado los mayores y menores porcentajes del total de respuestas facilitadas por los centros de la muestra.

\begin{tabular}{|l|c|}
\hline \multicolumn{2}{|c|}{ Alumnos con Dificultades de Aprendizaje } \\
\hline Evaluación de Capacidades y Aptitudes & $13,70 \%$ \\
\hline Evaluación de Actitudes e Intereses del alumno & $12,18 \%$ \\
\hline Información Académica y Vocacional & $9,65 \%$ \\
\hline Información sobre las posibilidades de formación no reglada/no formal & $9,14 \%$ \\
\hline
\end{tabular}


En este caso, las actuaciones de Orientación Vocacional y Profesional que más se suelen llevar a cabo se concentran en las áreas de Diagnóstico Psicopedagógico e Información. Sin embargo, existen otras intervenciones de Orientación entre las diecisiete opciones de respuesta propuestas, que los centros de la muestra no suelen desarrollar con estos alumnos. Entre ellas se encuentran: Autoempleo (técnicas, estrategias, ...); Coordinación con empresas, instituciones y/o agentes laborales de la zona; Organización de actividades en el exterior (visitas a centros, organismos oficiales etc.).

\begin{tabular}{|l|r|}
\hline \multicolumn{2}{|c|}{ Futuros alumnos de Programas de Garantía Social } \\
\hline Información sobre las posibilidades de formación no reglada/no formal & $13,73 \%$ \\
\hline Información Académica y Vocacional & $8,82 \%$ \\
\hline Evaluación de Capacidades y Aptitudes & $8,82 \%$ \\
\hline Evaluación de Actitudes e Intereses del alumno & $8,82 \%$ \\
\hline
\end{tabular}

Observamos aquí una similitud en cuanto a las intervenciones, pero diferencias en la prioridad que se les concede. Las actuaciones de Diagnóstico, que antes ocupaban el primer lugar, ahora son señaladas en menor medida por los centros. Por otro lado, para estos alumnos, la menor proporción de respuestas se da en las intervenciones relacionadas con el Autoempleo; Coordinación con empresas y Formación para la elaboración de su propio proyecto formativo y/o profesional.

\begin{tabular}{|l|r|}
\hline \multicolumn{2}{|c|}{ Alumnos con Necesidades Educativas Especiales } \\
\hline Evaluación de Actitudes e Intereses del alumno & \multicolumn{1}{|c|}{$12 \%$} \\
\hline Evaluación de Capacidades y Aptitudes & $11,33 \%$ \\
\hline Información Académica y Vocacional & $9,33 \%$ \\
\hline Desarrollo del Autoconcepto & $9,33 \%$ \\
\hline Fomento de las Habilidades Sociales & $8,66 \%$ \\
\hline
\end{tabular}

El análisis de los resultados muestra que, aunque también predominan las áreas de actuación de Diagnóstico e Información Académica, se introducen respecto a los grupos anteriores dos actividades que estarían más ligadas al ámbito de la Orientación Personal. Lo cual muestra el interés de los Departamentos de Orientación por desarrollar programas destinados a favorecer el desarrollo personal y social de los alumnos con necesidades educativas especiales, lo que resulta básico para su integración plena en la escuela y en la sociedad. Para estos alumnos, las intervenciones orientadoras que se desarrollan en menor medida suelen referirse al ámbito de la Orientación Laboral: Técnicas de búsqueda de empleo; Autoempleo; Conocimiento de las exigencias y ofertas del mercado laboral. 


\begin{tabular}{|l|r|}
\hline \multicolumn{2}{|c|}{ Alumnos con Necesidades de Compensación Sociocultural } \\
\hline Toma de decisiones & $10,59 \%$ \\
\hline Evaluación de Capacidades y Aptitudes & $10,59 \%$ \\
\hline Evaluación de Actitudes e Intereses del alumno & $9,41 \%$ \\
\hline Información sobre las posibilidades de formación no reglada/no formal & $9,41 \%$ \\
\hline Información Académica y Vocacional & $9,41 \%$ \\
\hline
\end{tabular}

Podemos apreciar aquí la aparición de la intervención en Toma de Decisiones como prioritaria en la Orientación de estos alumnos. Las actividades de Orientación que se desarrollan en menor medida con estos alumnos difieren en poco de las mencionadas para otros grupos, y se refieren, por consiguiente, al ámbito de la Orientación Profesional y Laboral: Conocimiento de las ofertas del mercado laboral; Técnicas de búsqueda de empleo y Autoempleo; Formación para la elaboración de su propio proyecto formativo y profesional.

\begin{tabular}{|l|c|}
\hline Alumnos con Dificultades de Adaptación Socioafectiva \\
\hline Desarrollo del Autoconcepto & $15,38 \%$ \\
\hline Evaluación de Actitudes e Intereses & $13,85 \%$ \\
\hline Evaluación de Capacidades y Aptitudes & $12,31 \%$ \\
\hline Fomento de las Habilidades Sociales & $12,31 \%$ \\
\hline
\end{tabular}

Las intervenciones que no se suelen realizan con estos alumnos coinciden con las que acabamos de comentar para los alumnos de Minorías Étnicas, relacionadas fundamentalmente con la Orientación Laboral.

\section{Conclusiones}

En primer lugar, hemos constatado en este trabajo, el impulso que ha experimentado el desarrollo de la Orientación en los últimos años, fundamentalmente a partir de la promulgación de la L.O.G.S.E. La institucionalización de los D.O. en Educación Secundaria ha contribuido a este desarrollo, pero consideramos que su fortalecimiento debería ir acompañado de una mayor estabilidad del Psicopedagogo en el mismo centro durante un período más prolongado de tiempo. Entendiendo la figura del Psicopedagogo u Orientador como el pilar sobre el que se organiza la intervención del Departamento de Orientación en el centro, pensamos que su continuidad en un mismo centro facilitaría el complejo proceso de planificación, desarrollo y puesta en marcha que supone introducir en la dinámica escolar la intervención orientadora.

Encontramos, por otra parte, que la creación de D.O. en todos los centros de Educación Secundaria, no ha llevado aparejada consigo, tal y como propone la normativa, un esfuerzo paralelo de dotación de recursos personales indispensables para la adecuada organización y funcionamiento de este Departamento, realidad apuntada en otros estudios (Royo, 2002). 
Consideramos que los componentes que forman parte de los D.O. de los centros de la muestra, no se corresponden con la oferta educativa que se imparte en los mismos, ni con las funciones asignadas al propio Departamento. Es decir, no existen los Profesores de Ámbito, ni los Maestros de Apoyo a la Integración que serían necesarios para que las enseñanzas que se imparten alcanzaran cierto nivel de calidad y ofrecieran una respuesta apropiada a las necesidades del alumnado que atienden. Creemos que la generalización de este profesorado en los D.O. puede contribuir a un mayor alcance, efectividad y sistematización de la intervención orientadora en Educación Secundaria.

En casi la totalidad de los centros se atiende a alumnos con necesidades educativas de algún tipo, pero la mayoría de los centros no disponen de la estructura ni los profesionales que las disposiciones legales establecen para atender adecuadamente esa diversidad. Encontramos, por tanto, una incoherencia entre lo que la normativa regula como necesario y lo que realmente pasa en los centros. Para que la Atención a la Diversidad fuera una realidad diaria en los centros de Educación Secundaria, sería necesario generalizar la presencia tanto de los Maestros especialistas de Apoyo a la Integración, como del profesorado que debe atender los P.D.C. y los P.G.S. (Maestro de Formación Básica, Apoyo a los Ámbitos, Formación y Orientación Laboral).

En cuanto a las Actuaciones de Orientación planificadas por el D.O., creemos que la diferenciación que se establece entre $1^{\text {er }}$ y $2^{\circ}$ ciclo de Educación Secundaria Obligatoria tiene su razón de ser en las diferentes necesidades que plantea este alumnado, atendiendo tanto a su situación evolutiva como educativa. Esto no contradice el principio de potenciación de la Orientación a todos los alumnos que marca la normativa, sino que favorece el desarrollo de la atención a la diversidad de necesidades, intereses, motivaciones, expectativas que presentan los alumnos que atraviesan por distintos momentos, desde el punto de vista educativo y vocacional. Defendemos la idea de un Orientación Profesional continua a lo largo de toda la escolaridad, pero no podemos obviar que la intervención sobre determinados aspectos, como la Toma de Decisiones, deben ser prioritarios en momentos determinados del proceso educativo.

Si nos centramos en las actuaciones de Orientación Vocacional y Profesional que se desarrollan con los a.c.n.e.e., pensamos que un paso positivo reside en reconocer que se está desarrollando alguna actividad concreta desde el D.O. para satisfacer las necesidades de dichos alumnos. Tras esta actitud positiva, encontramos, sin embargo, cierta reticencia a abandonar las tareas de diagnóstico y clasificación que han predominado durante mucho tiempo en la intervención desde la Orientación con estos alumnos. Nos alegra constatar que se está empezando a intervenir en el área de la Orientación para el Desarrollo Personal y Social (Desarrollo del Autoconcepto, Fomento de las Habilidades Sociales). Estas actuaciones pueden ser consideradas como un punto de partida para favorecer la integración y desarrollo de los alumnos diversos. Consideramos que se ha de potenciar la intervención con estos alumnos en el ámbito de la Orientación Profesional y Laboral, si nuestra intención es favorecer la plena integración de estos alumnos, no sólo en el sistema educativo, sino en el entorno social, como una tarea prioritaria desde la Orientación.

En recientes encuentros científicos ha quedado patente la necesidad de superar la función correctiva de la Orientación que, según nos muestran estos resultados y otros trabajos en la misma línea (Díaz Allué y otros, 1997; Rodicio, 2001), aún ocupa gran parte de la intervención psicopedagógica que se desarrolla en los centros educativos. Se han de abrir vías 
de trabajo que, basadas en los principios de prevención y de intervención social, concedan una mayor relevancia a otras funciones más integradoras de la Orientación, entendida como Intervención Psicopedagógica.

\section{Referencias bibliográficas}

Álvarez, M. y Fernández, R. (1996) (5ª ed.): Programas de orientación de estudios y vocacional al término de la escolaridad obligatoria (12-16). Recursos para su puesta en práctica. Barcelona: EUB.

Álvarez, M. y Rodríguez, S. (2000): Cambios socio-educativos y Orientación en el siglo XXI: Nuevas estructuras, roles y funciones. Actas del XII Congreso Nacional y I Iberoamericano de Pedagogía. Madrid: Sociedad Española de Pedagogía.

Arnal, J.; Rincón, D. y Latorre, A. (1994): Investigación educativa: fundamentos y metodologías. Barcelona: Labor.

Babío, M. (1996): «El asesor psicopedagógico en el Departamento de Orientación de un centro escolar». En C. Monereo e I. Solé (Coords.) El asesoramiento psicopedagógico: una perspectiva profesional y constructivista. Madrid: Alianza Editorial.

Bisquerra, R. (Coord.) (1998): Modelos de orientación e intervención psicopedagógica. Barcelona: Ed. Praxis.

Blanco, N. (1995): «La enseñanza secundaria obligatoria en una sociedad democrática». En J. Fernández Sierra (Coord.) El trabajo docente y psicopedagógico en Educación Secundaria. Málaga: Ediciones Aljibe.

Buendía, L.; Colás, P. y Hernández, F. (1997): Métodos de Investigación en Psicopedagogía. Madrid: McGraw-Hill.

Circular de 30 de Abril de 1996 de la Dirección General de Renovación Pedagógica por la que se dictan las Instrucciones sobre el Plan de Actividades de los Departamentos de Orientación de los Institutos de Educación.

Díaz Allué, M. T.; Carballo Santaolalla, R.; Fernández Díaz, M. J. y García Nieto, N. (1997): «Orientación en Educación Secundaria. Situación actual y prospectiva». Revista de Investigación Educativa, vol. 15, 2, 9-83.

Esteve, J. M. (2000): «El profesor de secundaria del siglo XXI. Un nuevo perfil para una nueva etapa de la educación». Actas del XII Congreso Nacional y I Iberoamericano de Pedagogía. Madrid: Sociedad Española de Pedagogía.

Fierro, A. (1985): «Desarrollo social y personalidad en la adolescencia». En M. Carretero; J. Palacios y A. Marchesi (Comps.) Psicología Evolutiva. Vol. III. Adolescencia, Madurez y Senectud. Madrid: Alianza.

Guerrero, J. F. (1995): «El papel del psicopedagogo ante las necesidades educativas especiales en Secundaria». En J. Fernández Sierra (Coord.) El trabajo docente y psicopedagógico en Educación Secundaria. Málaga: Ediciones Aljibe.

Hernández Pina, F; Fuentes Pérez, P.; Iglesias Verdegay, E. y Serrano Pastor, F. J. (1995): Introducción al proceso de investigación en educación. Murcia: DM-PPU.

Hortelano, M. A. y Jorge, J. (1997): El departamento de orientación. Guía y documentos para su quehacer curricular y psicopedagógico. Salamanca: Amarú Ediciones.

Ley 14/1970, de 4 de Agosto, General de Educación y de Financiación de la Reforma Educativa (B.O.E. 6 de Agosto de 1970). 
Ley Orgánica 1/1990, de 3 de octubre de Ordenación General del Sistema Educativo (B.O.E., 4 de octubre).

López Melero, M. (1995): «Diversidad y Cultura. Una escuela sin exclusiones». Kikirikí, 38, 26-38.

Proyecto para la Reforma de la Enseñanza. Propuesta para Debate (1987). MEC.

Resolución de 29 de abril de 1996 de la Dirección General de Centros Escolares sobre Organización de los Departamentos de Orientación en los Institutos de Educación Secundaria (B.O.E. 31 de Mayo de 1996).

Rodicio, M. L. (Coord.) (2001): «Simposio La Orientación en Galicia». Revista de Investigación Educativa, vol. 19, 2, 545-560.

Royo, F. (2002): «Desempeño profesional de los orientadores en los Institutos de Educación Secundaria de Salamanca. Creación de un instrumento de valoración y autovaloración». Revista Española de Orientación y Psicopedagogía, vol. 13, 1, 31-50.

Vélaz de Medrano, C. (1998): Orientación e intervención psicopedagógica. Concepto, modelos, programas y evaluación. Málaga: Ediciones Aljibe.

Fecha de recepción: 02-09-02

Fecha de revisión: 22-05-03

Fecha de aceptación: 27-10-03 